\title{
Respiratory variation in carotid peak systolic velocity predicts volume responsiveness in mechanically ventilated patients with septic shock: a prospective cohort study
}

Miguel Á Ibarra-Estrada ${ }^{1,2^{*}}$, José A López-Pulgarín ${ }^{1,2}$, Julio C Mijangos-Méndez ${ }^{1,2}$, José L Díaz-Gómez ${ }^{3}$ and Guadalupe Aguirre-Avalos ${ }^{1,2}$

\begin{abstract}
Background: The evaluation of fluid responsiveness in patients with hemodynamic instability remains to be challenging. This investigation aimed to determine whether respiratory variation in carotid Doppler peak velocity $(\triangle \mathrm{CDPV})$ predicts fluid responsiveness in patients with septic shock and lung protective mechanical ventilation with a tidal volume of $6 \mathrm{ml} / \mathrm{kg}$.

Methods: We performed a prospective cohort study at an intensive care unit, studying the effect of 59 fluid challenges on 19 mechanically ventilated patients with septic shock. Pre-fluid challenge $\triangle C D P V$ and other static or dynamic measurements were obtained. Fluid challenge responders were defined as patients whose stroke volume index increased more than $15 \%$ on transpulmonary thermodilution. The area under the receiver operating characteristic curve (AUROC) was compared for each predictive parameter.

Results: Fluid responsiveness rate was $51 \%$. The $\triangle$ CDPV had an AUROC of 0.88 (95\% confidence interval (CI) $0.77-0.95)$; followed by stroke volume variation $(0.72,95 \% \mathrm{Cl} 0.63-0.88)$, passive leg raising $(0.69,95 \% \mathrm{Cl}$ $0.56-0.80)$, and pulse pressure variation $(0.63,95 \% \mathrm{Cl} 0.49-0.75)$. The $\triangle$ CDPV was a statistically significant superior predictor when compared with the other parameters. Sensitivity, specificity, and positive and negative predictive values were also the highest for $\triangle C D P V$, with an optimal cutoff at $14 \%$. There was good correlation between $\triangle$ CDPV and SVI increment after the fluid challenge $(r=0.84 ; p<0.001)$.

Conclusions: $\triangle C D P V$ can be more accurate than other methods for assessing fluid responsiveness in patients with septic shock receiving lung protective mechanical ventilation. $\triangle$ CDPV also has a high correlation with SVI increase after fluid challenge.
\end{abstract}

Keywords: Sepsis; Carotid Doppler peak velocity; Pulse pressure variation; Passive leg raising; Transpulmonary thermodilution

\footnotetext{
* Correspondence: drmiguelibarra@hotmail.com

"Intensive Care Unit, Hospital Civil de Guadalajara "Fray Antonio Alcalde",

Hospital 278, El Retiro, Specialties Building, Floor 1, Guadalajara, Jalisco 44280,

Mexico

${ }^{2}$ Centro Universitario de Ciencias de la Salud, Universidad de Guadalajara,

Independencia Oriente, 44340 Guadalajara, Jalisco, Mexico

Full list of author information is available at the end of the article
} 


\section{Background}

In a patient with acute hemodynamic instability, a fluid challenge will cause an increase in stroke volume, according to the Frank-Starling curve [1]. This increase in stroke volume has a salutary effect because it improves tissue perfusion. In contrast, higher hydrostatic pressures in the vascular system predispose the patient to edema, organic dysfunction, and increased risk of inhospital mortality [2,3]. Relative hypovolemia has been described in the setting of septic shock. However, only $50 \%$ of patients with hemodynamic instability are fluid responsive $[4,5]$. Therefore, expeditious fluid resuscitation is advised, and clinicians must always weigh the benefits and risks of intravenous fluids [2, 6].

Currently, both static and dynamic parameters are utilized for prediction of fluid responsiveness. Static parameters (e.g., central venous pressure and pulmonary artery occlusion pressure) are much less reliable than dynamic parameters, which are based on respirophasic variation in stroke volume (e.g., pulse pressure variation and changes in aortic blood flow) [7]. Most common dynamic parameters are invasive (arterial and/or central venous cannulation is required) and expensive. Echocardiography is a well-established method for evaluating fluid responsiveness [5, 8, 9]. Nevertheless, measurement of left ventricular outflow tract velocities for the estimation of stroke volume is labor intense, requires specific training for adequate performance, and is not easily reproducible or obtainable $[8,10]$. Thus, alternative methods, including brachial or carotid artery velocity, have been examined as surrogates for stroke volume in the non-septic shock patient population [11, 12]. Moreover, most predictive indices for volume responsiveness are not validated in patients receiving lung protective ventilatory strategies. The aim of this study was to determine if respiratory variation in carotid Doppler peak velocity $(\triangle \mathrm{CDPV})$ can predict fluid responsiveness in patients with septic shock and lung protective mechanical ventilation.

\section{Methods}

\section{Patients}

This was a single-center, prospective, cohort study. Inclusion criteria were mechanical ventilation, septic shock, and hemodynamic instability for which the attending intensivist determined the need for fluid challenge based on signs of inadequate tissue perfusion according to Surviving Sepsis Campaign recommendations [13]. The investigation was conducted in a medical/surgical intensive care unit and tertiary academic hospital from May 2014 through October 2014. Exclusion criteria were age under 18 years, non-septic origin of shock, known heart failure, valvular disease or arrhythmia, intra-abdominal hypertension, peripheral arterial disease, common carotid artery stenosis greater than $50 \%$ (systolic peak velocity $>182 \mathrm{~cm} / \mathrm{s}$ and/or diastolic velocity $>30 \mathrm{~cm} / \mathrm{s}$ by Doppler ultrasound), spontaneous respiratory efforts, and utilization of colloids other than albumin for the fluid challenge [14]. Volume controlled mechanical ventilation was performed with tidal volumes at $6 \mathrm{ml} / \mathrm{kg}$ of predicted body weight. We usually administer fluid challenges with normal saline at a $7 \mathrm{~mL} / \mathrm{kg}$ dose over a 30-min period and perform thermodilution before and after each challenge. The Institutional Review Board at Hospital Civil de Guadalajara deemed the investigation to be of minimal risk and waived the need for written consent.

\section{Measurements and volume responsiveness}

Before each fluid challenge, carotid peak systolic velocity was measured with a Micromaxx System (Sonosite, WA, USA), using a $5-10-\mathrm{mHz}$ linear array transducer. After procuring a longitudinal view of the common carotid artery, pulsed Doppler analysis at $2 \mathrm{~cm}$ from the bifurcation was performed. The sample volume was positioned at the center of the vessel, with angulation at no more than $60^{\circ}$. Maximum and minimum peak systolic velocities were obtained in a single respiratory cycle (Fig. 1), and the $\triangle \mathrm{CDPV}$ was calculated with the following formula: (MaxCDPV - MinCDPV) / [(MaxCDPV + MinCDPV) / 2] $\times 100$, expressed as a percentage [12] Two investigators with previous formal training in critical care ultrasound estimated the $\triangle \mathrm{CDPV}$. These investigators were blinded to each other's results and to all other variables. The mean of both measurements obtained by the two investigators was used. In addition, the same investigators evaluated the adequate procurement of transthoracic echocardiographic windows for estimation of the stroke volume. Pulse pressure variation (PPV) was calculated with the formula: PPV $(\%)=100 \times$ (Pp max - Pp min) / [(Pp max + Pp min) / 2] [15], with pressures measured from a femoral arterial catheter with the v2.6e monitor (Phillips Healthcare, Eindhoven, the Netherlands). The passive leg raising (PLR) test was performed as previously reported [16] before each challenge by placing the patient's head and upper torso upright at $45^{\circ}$. This was followed by a flat supine position and raising both legs to a $45^{\circ}$ angle from the bed, while measuring the SVI before and after the maneuver. The highest SVI from the first $3 \mathrm{~min}$ after the test was taken, and the percentage increase in SVI with the PLR was recorded. Inferior vena cava diameter (IVC-d) measurement was performed with a two-dimensional view at a subxyphoidal long axis, approximately $2 \mathrm{~cm}$ caudal to the hepatic vein inlet. Maximum and minimum diameters over a single respiratory cycle were recorded, and respiratory variation in inferior vena cava diameter ( $\triangle \mathrm{D}$-IVC) was calculated with the formula: (Max 


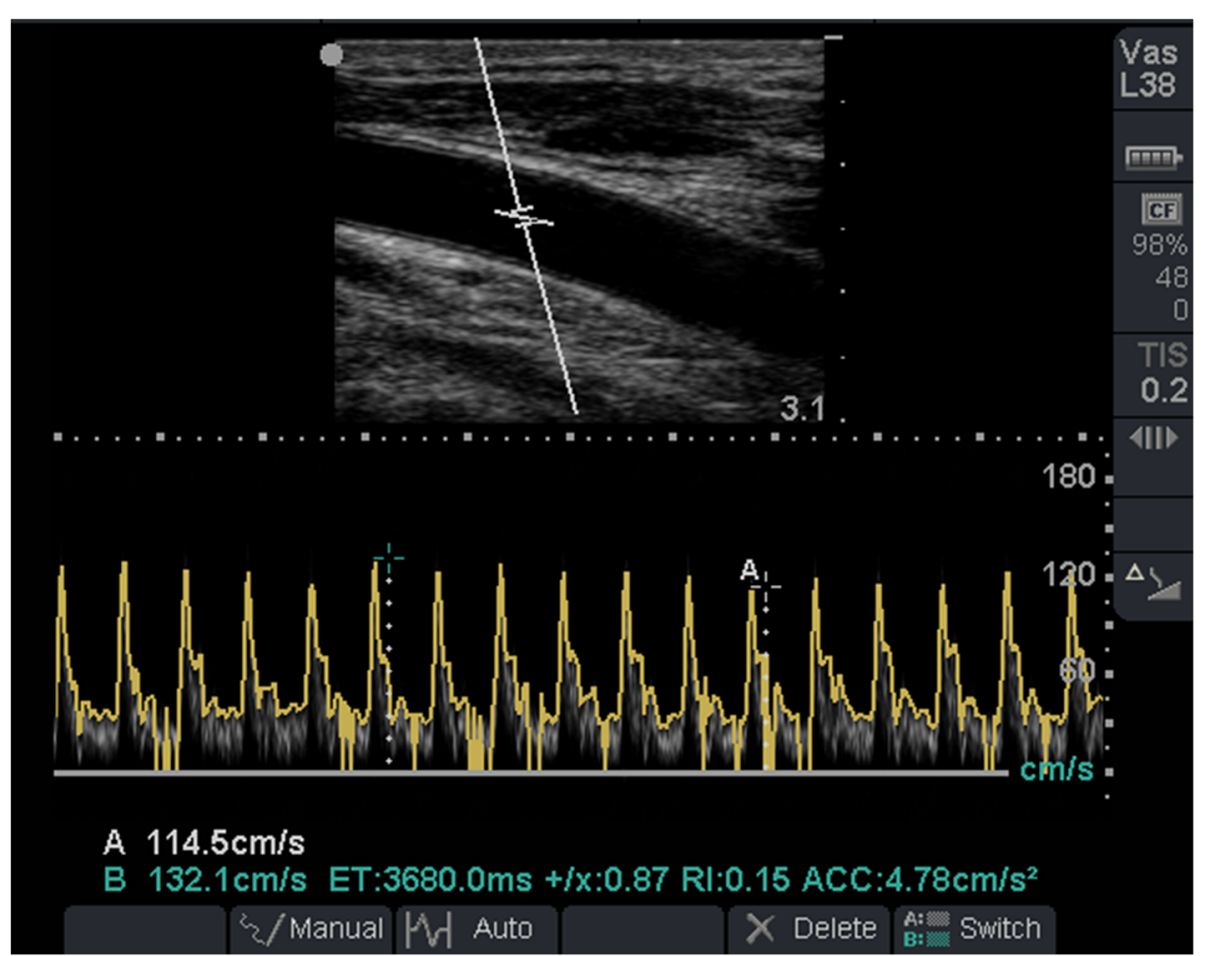

Fig. 1 Measurement of variation in carotid peak systolic velocity. At $14 \%$ in this patient

IVC-d - Min IVC-d) / [(Max IVC-d + Min IVC-d) / 2]. Transpulmonary thermodilution was performed before and after each fluid challenge with the Pulse Contour Cardiac Output system (Pulsion Medical Systems, Münich, Germany) to obtain an automated SVI, stroke volume variation (SVV), and other variables. Patients with an increase of more than $15 \%$ in the SVI after the fluid challenge were classified as "responders", and those with an increase of less than $15 \%$ in the SVI or those with no increase were classified as "non-responders."

\section{Statistical analysis}

Continuous variables were reported as the mean (standard deviation) if they were normally distributed or the median (interquartile range) if they were not normally distributed, using the Shapiro-Wilk test. Preload indices were compared in responders and non-responders using the Mann-Whitney test. Categorical variables were expressed as the number of measurements (\%) and were compared by the chi-squared test. For analyzing the trend in response at repeated fluid challenges per patient, we used the Cochran-Armitage test. We constructed receiver operating characteristic (ROC) curves for static and dynamic indices of preload to determine the ability to predict fluid responsiveness, and their area under the curve was compared using the Hanley-McNeil test [17]. Optimal cutoff values were obtained with the greatest sum of sensitivity and specificity using the Youden index [18]. The relationship between preload indices and changes in SVI after the fluid challenge was estimated with Spearman's correlation coefficient test. We determined inter-observer reproducibility for $\triangle \mathrm{CDPV}$ by using the Bland-Altman Plot, described as mean bias [19]. Inter-rater agreement was calculated with the kappa statistic and a $95 \%$ confidence interval (CI) [20]. Assuming a fluid responsiveness rate of $50 \%$, we determined that 36 measurements would be needed to detect differences of 0.30 between the area under the receiver operating characteristic (AUROC) curve of central venous pressure (0.55) [4] and $\triangle \mathrm{CDPV}(0.85)$ [11], with an $80 \%$ power and type I error of $5 \%$. For all tests, $p$ values were two-sided, and a $p$ value lower than 0.05 was considered statistically significant. We used MedCalc (Ver 13.2, Mariakerke, Belgium) for calculating the sample size and for the statistical analysis.

\section{Results}

A total of 59 fluid challenges were performed in 19 patients, with a responsiveness rate of $51 \%$. In eight patients $(40 \%)$, the velocity-time integral at the left ventricle outflow tract was not obtained due to an unfavorable transthoracic echocardiographic window. Baseline characteristics of the patients are shown in Table 1. 
Table 1 Characteristics and baseline parameters before all fluid challenges, between responders and non-responders

\begin{tabular}{|c|c|c|c|}
\hline \multirow[t]{2}{*}{ Variable } & \multirow{2}{*}{$\begin{array}{l}\text { Responders } \\
n=30\end{array}$} & \multirow{2}{*}{$\begin{array}{l}\text { Non-responders } \\
n=29\end{array}$} & \multirow[t]{2}{*}{$P$ value } \\
\hline & & & \\
\hline Age (years) & $51(38-58)$ & $53(38-57)$ & 0.65 \\
\hline Gender (male, \%) & $20(67)$ & $17(59)$ & 0.71 \\
\hline $\mathrm{BSA}\left(\mathrm{m}^{2}\right)$ & $1.65(1.50-1.80)$ & $1.60(1.57-1.80)$ & 0.72 \\
\hline ARDS (\%) & $22(73)$ & $21(72)$ & 0.83 \\
\hline AKI (\%) & $15(50)$ & $14(48)$ & 0.88 \\
\hline Shock diagnosis (hours) & $17.5(13-23)$ & $27(21.7-35)$ & 0.0001 \\
\hline MAP (mmHg) & $61(60-62.8)$ & $63(61-64.2)$ & 0.053 \\
\hline HR (beats/min) & $119(117-123)$ & $121(115-124)$ & 0.63 \\
\hline$\cup O(\mathrm{ml} / \mathrm{kg} / \mathrm{h})$ & $0.4(0.2-0.6)$ & $0.4(0.2-0.8)$ & 0.34 \\
\hline Arterial Lactate $(\mathrm{mmol} / \mathrm{L})^{\mathrm{a}}$ & $5.3 \pm 1.6$ & $4.7 \pm 1.7$ & 0.20 \\
\hline ScvO2 (\%) & $64 \pm 10.7$ & $67 \pm 10.2$ & 0.26 \\
\hline $\mathrm{SVI}\left(\mathrm{ml} / \mathrm{m}^{2}\right)$ & $16(14-18)$ & $17(15-19)$ & 0.09 \\
\hline NE dose (mcg/kg/min) & $0.33(0.21-0.57)$ & $0.40(0.33-0.54)$ & 0.36 \\
\hline Fluid balance $(L)^{a}$ & $2.12 \pm 0.70$ & $2.62 \pm 0.75$ & 0.01 \\
\hline Fluid loads (n) & $2(2-3)$ & $2(2-3)$ & 0.19 \\
\hline SOFA (score) & $10(8-13)$ & $15(13-18)$ & $<0.0001$ \\
\hline $\operatorname{PEEP}\left(\mathrm{cm} / \mathrm{H}_{2} \mathrm{O}\right)$ & $6(5-7)$ & $6(5-7)$ & 0.71 \\
\hline Plateau pressure $\left(\mathrm{cm} / \mathrm{H}_{2} \mathrm{O}\right)^{\mathrm{a}}$ & $24 \pm 3$ & $24 \pm 3$ & 0.98 \\
\hline Tidal volume (ml/kg) & $6(6.0-6.3)$ & $6(6.0-6.3)$ & 0.96 \\
\hline
\end{tabular}

All data are expressed as median (interquartile range), except those marked with ${ }^{a}$, which are expressed as mean (standard deviation)

$B S A$ body surface area, ARDS acute respiratory distress syndrome, AKI acute kidney injury, MAP mean arterial pressure, $H R$ heart rate, UO urinary output, $\mathrm{SCVO} 2$ oxygen saturation at central venous blood, SVI stroke volume index, NE norepinephrine, SOFA Sequential Organ Failure Assessment score, range from 0 to 24 , with higher scores indicating a greater risk of mortality, PEEP positive end-expiratory pressure

\section{Predictors of fluid responsiveness}

The $\triangle$ CDPV, SVV, SVI increment following the PLR test, and PPV were significantly higher in responders than in non-responders. There was no significant difference in the $\triangle \mathrm{D}$-IVC or in any of the static parameters (Table 2). Among dynamic variables, $\triangle \mathrm{CDPV}$ had the highest AUROC (0.88, $p<0.001 ; 95 \%$ CI 0.77-0.95) (Table 3), with an optimum cutoff value of greater than $14 \%$ based on the Youden index. Using the Hanley-McNeil test, $\triangle \mathrm{CDPV}$ was significantly superior to the other variables, $(p=0.03$ versus SVV, $p=0.01$ versus PLR, $p=0.001$ versus PPV, and $p<0.001$ versus $\triangle \mathrm{D}$-IVC; Fig. 2). $\triangle C D P V$ showed the highest sensitivity and specificity, as well as positive and negative predictive values (Table 4).

Because it may be arguable as to what cutoff points of increase in SVI is truly clinically meaningful, we calculated ROC curves of the main indices taking an increase in SVI $>10 \%$, instead of $>15 \%$ as cutoff. The results were similar, as $\triangle \mathrm{CDPV}$ maintained the greatest AUROC $(0.90, p=<0.001)$.
Table 2 Baseline differences in predictors of fluid responsiveness between responders and non-responders

\begin{tabular}{llll}
\hline Variable & $\begin{array}{l}\text { Responders } \\
n=30\end{array}$ & $\begin{array}{l}\text { Non-responders } \\
n=29\end{array}$ & $P$ value \\
\hline$\Delta$ CDPV (\%) & $21(17-23)$ & $13(10-14)$ & $<0.0001$ \\
SW (\%) & $18.5(17-22)$ & $14(11-18)$ & 0.003 \\
PLR Delta SVI (\%) & $16.5(13-18)$ & $13(10.5-16)$ & 0.01 \\
PPV (\%) & $14.5(11-19)$ & $12(10-14)$ & 0.08 \\
$\Delta$ D-IVC (\%) & $15(12-17)$ & $14(11-18)$ & 0.54 \\
GEDI $\left(\mathrm{ml} / \mathrm{m}^{2}\right)^{\mathrm{a}}$ & $472 \pm 115$ & $490 \pm 102$ & 0.53 \\
IVC-d $(\mathrm{mm})^{\mathrm{a}}$ & $10.9 \pm 3.8$ & $10.5 \pm 3.7$ & 0.69 \\
CVP $(\mathrm{mmHg})$ & $8(6-10)$ & $8(6-9)$ & 0.78 \\
\hline
\end{tabular}

Data are expressed as median (interquartile range), except those marked with ${ }^{a}$, which are expressed as a mean (standard deviation)

$\triangle C D P V$ respiratory variation in carotid Doppler peak velocity, SVV stroke volume variation, PLR Delta SVI rise in stroke volume index after passive leg elevation test, $P P V$ pulse pressure variability, ScVO2 oxygen saturation at central venous blood, $\triangle D-I V C$ respiratory variation in inferior vena cava diameter, GEDI global end-diastolic index, IVC- $d$ inferior vena cava diameter, CVP central venous pressure

Responders had a significantly higher median rise in the SVI after the fluid challenge compared to nonresponders ( 42 versus $9.3 \%, p<0.001$ ), notwithstanding pre-challenge SVI was not different $\left(16 \mathrm{ml} / \mathrm{m}^{2}\right.$ versus $\left.17 \mathrm{ml} / \mathrm{m}^{2}, p=0.09\right)$. As seen in Table 5, at repeated measures analysis, there was no significant trend in the progressive number of fluid challenges per patient and responsiveness rate $(p=0.29)$ or $\Delta \mathrm{CDPV}(p=0.32)$. Median in time between fluid challenges per patient was $4 \mathrm{~h}$ (IQR 3.2-5). The presence of acute respiratory distress syndrome or acute kidney injury was not associated with a lack of response to fluid challenge. There was no newly detected carotid stenosis or diminished ejection fraction. The mean time to obtain $\triangle \mathrm{CDPV}$ was $54 \mathrm{~s}$ (SD, $3.9 \mathrm{~s})$.

\section{Prediction of the hemodynamic effects of fluid challenge} Only $\triangle$ CDPV was positively correlated with a fluid challenge-induced change in the SVI, and $\triangle \mathrm{CDPV}$ had the highest correlation coefficient $(r=0.84, p<0.001$, $95 \%$ CI 0.74-0.90). A regression formula for predicting a rise in the SVI after fluid challenge was obtained (Fig. 3). The correlation between SVV and SVI increase due to fluid challenge was low $(r=0.24, p=0.058,95 \%$ CI $\left.-0.009-0.47, r^{2}=0.06\right)$. There was no significant correlation between the other indices and change in the SVI.

\section{Reproducibility and agreement of $\triangle \mathrm{CDPV}$}

Bland-Altman analysis showed good concordance between estimation of $\triangle \mathrm{CDPV}$ by the two investigators, with a mean bias of 0.2 and limits of agreement between -1.9 and 2.3 (Fig. 4). The inter-observer variability was good, with a kappa statistic of 0.87 (95\% CI 0.84-0.91). 
Table 3 Correlations between predictors and the fluid challenge-induced change in the stroke volume index

\begin{tabular}{lllll}
\hline Variable & Correlation coefficient $(95 \% \mathrm{Cl})$ & $p$ value & AUROC $(95 \% \mathrm{Cl})$ & $p$ value \\
\hline$\Delta$ CDPV (\%) & $0.84(0.74-0.90)$ & $<0.001$ & $0.88(0.77-0.95)$ & 0.001 \\
SW (\%) & $0.24(-0.009-0.47)$ & $0.05-0.83)$ & 0.001 \\
PLR Delta SVI (\%) & $0.24(-0.01-0.46)$ & 0.06 & $0.69(0.56-0.80)$ & 0.005 \\
PPV (\%) & $0.02(-0.23-0.27)$ & 0.08 & $0.63(0.49-0.75)$ & 0.007 \\
$\Delta$ D-IVC (\%) & $-0.02(-0.27-0.23)$ & 0.84 & $0.54(0.41-0.67)$ & 0.50 \\
GEDI (ml/m $\left.{ }^{2}\right)$ & $0.02(-0.23-0.27)$ & 0.85 & $0.55(0.41-0.68)$ & 0.48 \\
IVC-d (mm) & $0.04(-0.21-0.29)$ & 0.74 & $0.52(0.39-0.65)$ & 0.75 \\
CVP $(\mathrm{mmHg})$ & $-0.09(-0.34-0.16)$ & 0.48 & $0.52(0.38-0.65)$ & 0.78 \\
\hline AUROC a
\end{tabular}

$A U R O C$ areas under the receiver operating characteristic curves, $\triangle C D P V$ respiratory variation in carotid Doppler peak velocity, SVV stroke volume variation, $P L R$ Delta SVI rise in stroke volume index after passive leg elevation test, PPV pulse pressure variation, ScvO2 oxygen saturation at central venous blood, $\triangle D-I V C$ respiratory variation in inferior vena cava diameter, GEDI global end-diastolic index, IVC- $d$ inferior vena cava diameter, CVP central venous pressure

\section{Discussion}

The principal finding of this study is that $\triangle \mathrm{CDPV}$ is easily obtainable and more accurate than conventional methods (central venous pressure, respiratory variation in inferior vena cava diameter, pulse pressure variation) for assessing fluid responsiveness in mechanically ventilated patients with septic shock. Furthermore, the $\triangle \mathrm{CDPV}$ has a high correlation with SVI increase after fluid challenge. To our knowledge, this is the first investigation that utilizes the $\triangle \mathrm{CDPV}$ as a predictor of fluid responsiveness in patients with lung protective mechanical ventilation and septic shock.

In our study, up to $40 \%$ of the patient population had technically difficult echocardiographic apical views, which limited the measurement of the velocity time

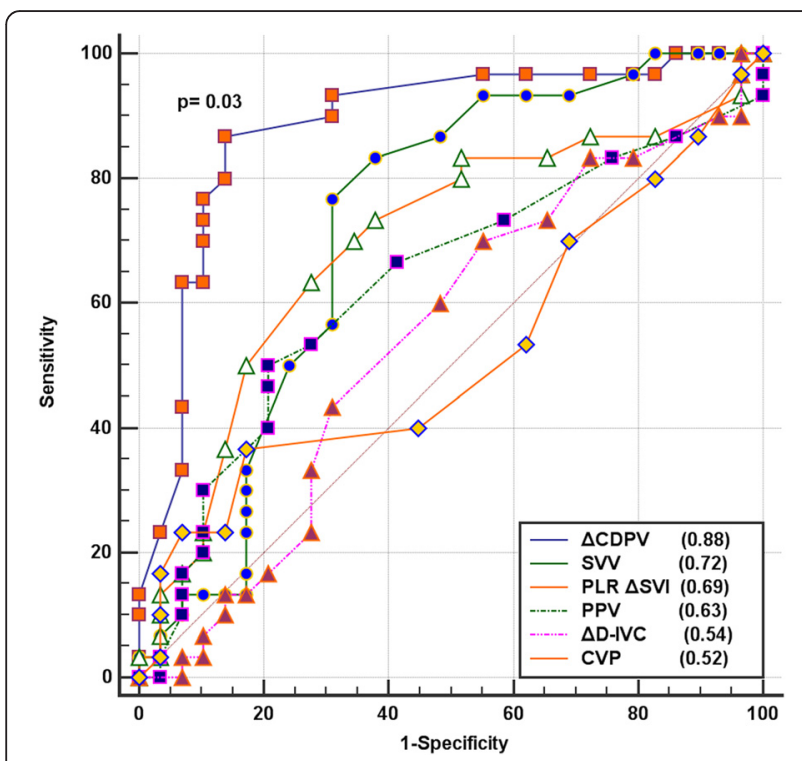

Fig. 2 Areas under the receiver operating characteristic curve of predictors of fluid responsiveness. The $p$ value indicates comparison between respiratory variation in carotid peak velocity and stroke volume variation (SW) with the Hanley-McNeil test integral at the left ventricle outflow tract. Hence, alternative non-invasive and practical methods for assessment of fluid responsiveness in septic shock should be investigated. This is a limitation of point-of-care echocardiography in the ICU because the procurement of different acoustic windows varies and presents different degrees of difficulty for mastery [21]. Moreover, Young et al. demonstrated that TTE failed to evaluate the ejection fraction in $69 \%$ of the patients in the ICU [22]. Furthermore, measurement of carotid peak flow can be rapidly performed with less difficulty than for other echocardiographic variables [16].

Other authors have explored the applicability of echocardiography in mechanically ventilated patients. Feissel et al. [23] reported high accuracy, sensitivity (100\%), and specificity (89\%) of respiratory variation in aortic blood velocity (cutoff value higher than $12 \%$ ) for prediction of fluid responsiveness in septic patients receiving mechanical ventilation. Similarly, Monnet et al. [24] showed the respiratory variation in aortic peak velocity (cutoff value higher than $13 \%$ ) as a predictor of fluid responsiveness with an AUROC of 0.82 and sensitivity and specificity of 80 and $72 \%$, respectively. However, these studies utilized an invasive method such as transesophageal echocardiography, and patients with an inadequate aortic blood flow signal were excluded. Moreover, not all patients had septic shock [24], and they were ventilated with tidal volumes greater than $6 \mathrm{~mL} / \mathrm{kg}[23,24]$.

Monge Garcia et al. [12] demonstrated that the variation in brachial artery peak velocity was a good predictor of fluid responsiveness, with a sensitivity and specificity of 74 and $95 \%$, respectively. The AUROC was 0.88 , similar to our method. In contrast to our study, only half of the patients in their study were septic, and they used the Flo Trac/Vigileo system. This system is a non-calibrated monitoring device for which the accuracy for tracking changes in the cardiac index has come under question. A recent study compared the arterial pressure waveform-derived cardiac index provided by 
Table 4 Cutoffs and diagnostic performances of significant predictors

\begin{tabular}{llllll}
\hline Variable & Cutoff & Sensitivity & Specificity & PPV $^{\text {a }}$ & NPV \\
$(95 \% \mathrm{Cl})$ & $(95 \% \mathrm{Cl})$ & $86(69-96)$ & $85(67-95)$ \\
\hline$\Delta$ CDPV (\%) & $>14$ & $86(69-96)$ & $86(68-96)$ & $71(52-85)$ & $73(52-88)$ \\
SW (\%) & $>16$ & $76(57-90)$ & $68(49-84)$ & $70(49-86)$ & $65(46-81)$ \\
PLR Delta SVI (\%) & $>15$ & $63(44-80)$ & $79(60-97)$ & $71(48-88)$ & $60(43-75)$ \\
PPV (\%) & $>14$ & $50(31-68)$ &
\end{tabular}

Only the indices with an AUROC $>0.6$ and $p<0.05$ were included. Cutoffs estimated by Youden index

NPV negative predictive value, $\triangle C D P V$ carotid peak velocity variation, SVV stroke volume variation, PLR Delta SVI rise in stroke volume index after passive leg elevation test, PPV pulse pressure variation

${ }^{\text {a }}$ Positive predictive value

the Vigileo system and pulse contour-derived cardiac index provided by the PiCCO device. The former device performed poorly, with lack of response to therapeutic interventions (volume expansion and vasopressor administration). We used the PiCCO system in this investigation [25].

The preferential diversion of blood flow toward the carotid arteries, away from the peripheral arteries, is a relevant pathophysiological consideration in patients suffering from shock [26]. Considering these facts as well as the flaws in radial artery-based monitoring [27, 28], Song et al. [11] evaluated peak velocity variation at the carotid artery. They showed an AUROC of 0.85, with a threshold value for fluid responsiveness of $11 \%$ (sensitivity and specificity of 0.83 and 0.82 ). These results are similar to our study. However, their study population primarily consisted of coronary artery disease patients. In comparison to Song's study, we showed a higher correlation between $\triangle \mathrm{CDPV}$ and a fluid challenge-induced SVI increase $(r=0.84$ versus $r=0.63)$ [11]. This finding could be explained by the higher mean age in their study. Perhaps their patients have lower vessel compliance and/or reduced cardiac reserve with concomitant coronary artery disease.

Recently, Marik et al. [26] evaluated the blood flow changes in the carotid artery after a PLR maneuver as a predictor of fluid responsiveness in 34 hemodynamically unstable patients. Among these patients, $65 \%$ presented with severe sepsis/septic shock, and $56 \%$ required mechanical ventilation. The increase in carotid blood flow of greater than or equal to $20 \%$ after PLR was found to have a sensitivity and specificity of 94 and $86 \%$, respectively. The AUROC curve was, however, not estimated. Their method differed from our study because they not only measured the systolic peak velocity but also calculated the variation in blood flow, which is more labor intensive because it requires measurement of the vessel diameter. In addition, they coupled Doppler estimations with a PLR maneuver. In contrast, we obtained an adequate discriminatory performance with a simpler method, using only the peak systolic velocities at a single respiratory cycle, which showed good interobserver agreement and reproducibility.

There has been a recent interest in the response to fluid administration over time in patients with shock. Nunes et al. [29] highlighted the limited success of volume resuscitation in patients with circulatory shock after initial resuscitation $(>6 \mathrm{~h}$ ). Thus, a fluid challenge response is not always sustained. We found similar results, as the time from diagnosis of septic shock had a median of $22 \mathrm{~h}$. Another relevant finding in the aforementioned study is that after a fluid challenge, the cardiac index with crystalloid (500 $\mathrm{mL}$ infused over a 30-min period) decreased toward baseline values $60 \mathrm{~min}$ after infusion, even in responders. Hence, we performed our analysis with the number of "measurements" rather than the number of "patients." Most patients, who are classified as responders, could have received additional fluid challenges at any time for different clinical contexts (e.g., fluid balance and challenges, presence of organic failure, and different vasopressor dosages).

The performance of PPV and SVV for prediction of fluid responsiveness has been previously reported, with both sensitivity and specificity higher than $90 \%[15,30]$. However, in our study, its predictive accuracy was lower than expected. A possible explanation for this difference

Table 5 Repeated measures analysis

\begin{tabular}{|c|c|c|c|c|c|c|}
\hline & \multicolumn{5}{|c|}{ Fluid challenge (\#) } & \multirow[t]{2}{*}{$p$ value for trend ${ }^{a}$} \\
\hline & $\# 1$ & $\# 2$ & $\# 3$ & $\# 4$ & \#5 & \\
\hline Response (n) & 11 & 9 & 7 & 2 & 1 & 0.29 \\
\hline No-response $(n)$ & 8 & 8 & 8 & 3 & 2 & \\
\hline$\Delta \mathrm{CDPV} \%(\mathrm{SD})$ & $19.4(6.7)$ & $16.4(7.4)$ & $14.8(6.3)$ & $14.5(6.1)$ & $16.3(4.9)$ & 0.32 \\
\hline
\end{tabular}

${ }^{a}$ Cochran-Armitage test for response/no-response. Repeated measures ANOVA for $\triangle$ CDPV 


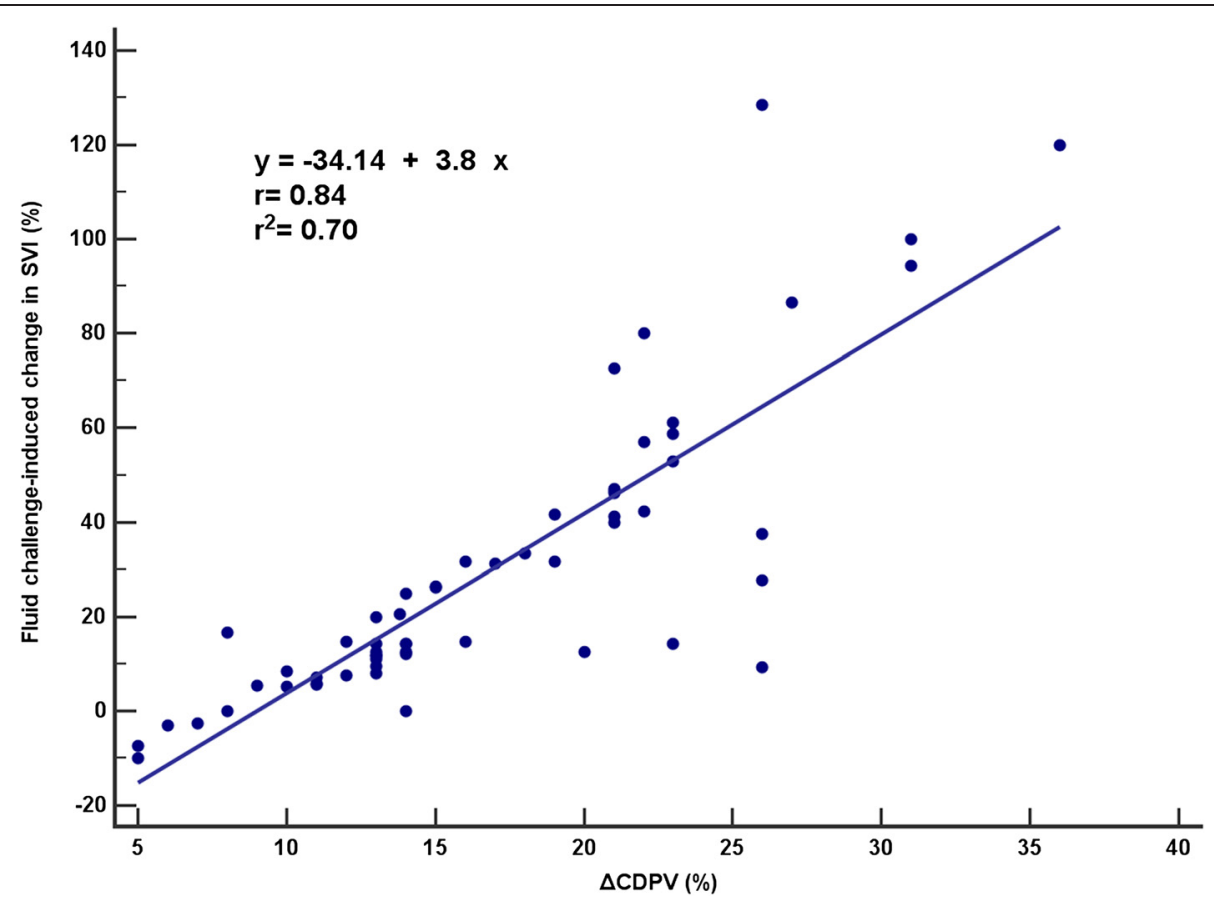

Fig. 3 Correlation between variation in respiratory carotid peak systolic velocity and fluid challenge-induced changes in the stroke volume index

could be the aforementioned preferential diversion of blood flow toward the carotid arteries and away from the peripheral arteries [26], as well as that the fluid challenges in the study were administrated to mechanically ventilated patients with tidal volumes greater than or equal to $8 \mathrm{~mL} / \mathrm{kg}$ [15], whereas this variable is not specified in the other studies [31, 32]. We followed a lung protective ventilator strategy with tidal volumes of $6 \mathrm{~mL} / \mathrm{kg}$ in all patients [33], and high tidal volume influences the hemodynamic effects of a fluid challenge [34].

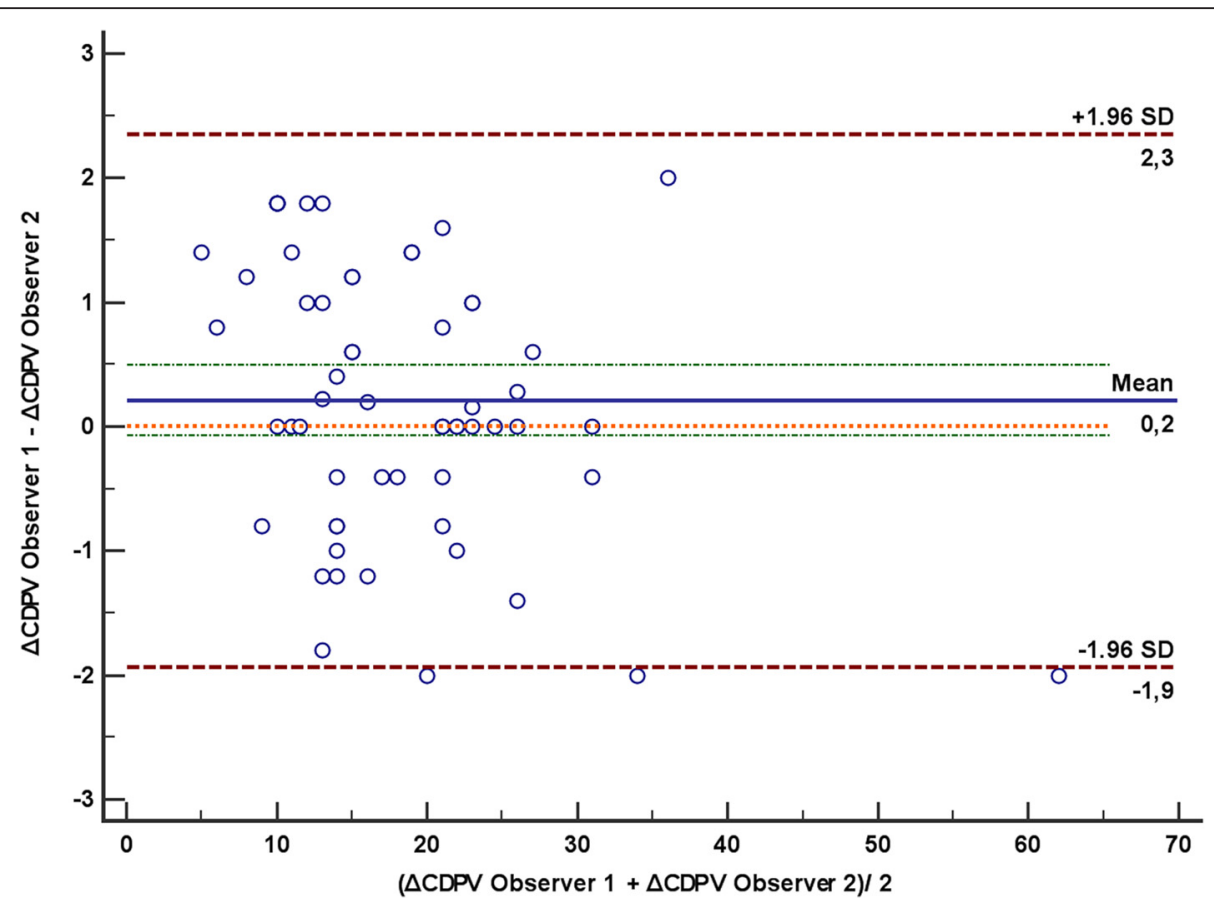

Fig. 4 Bland-Altman plot for measurements of both observers. There was a mean bias of 0.2 , with limits of agreement between -1.9 and 2.3 


\section{Limitations}

This study has some limitations. As a respirophasic dynamic index, $\triangle \mathrm{CDPV}$ does not apply to patients with spontaneous breathing, arrhythmias, valvular disorders, significant heart failure, and common carotid stenosis. Nonetheless, measurement of $\triangle \mathrm{CDPV}$ is a reliable method, without the inherent risk of central artery cannulation, which is present for thermodilution or pulse contour analysis systems. Additionally, there is no need to raise the patient's legs, which is a time-consuming maneuver. Further, it is discouraged and/or unreliable in postsurgical, abdominal hypertension, or fractured patients. We performed point-of-care echocardiography to address ejection fraction in all patients. However, we did not record the ratio of early transmitral flow velocity to the early diastolic velocity of the mitral annulus (E/E'); therefore, the incidence of elevated left ventricular filling pressures was unknown. This could be a possible bias in the study, as an elevated E/E' $(>15)$ is negatively correlated with lower performance at prediction of fluid responsiveness $[35,36]$. In order to minimize time, we performed carotid measurements on a single respiratory cycle; therefore, we do not know if the accuracy could have been improved with the average of three respiratory cycles.

Although the reliability of $\mathrm{PiCCO}$ system has been found to be good in heterogeneous groups of patients, it is still questioned, mainly on the time-dependent accuracy on recalibrations and its variability on agreement at different vasopressor dose [30, 37, 38]. These data were not addressed in our study.

External validity is limited, as physicians involved at estimation of $\triangle \mathrm{CDPV}$ were trained in critical care sonography for more than 1 year. Also, due to the observational nature of the study, management was not $\triangle \mathrm{CDPV}$-guided, and the spectrum of patients was narrow, including septic shock patients only. As long as there are clinical trials addressing these issues, our results should be interpreted cautiously. For statistical comparison between ROC curves with the Hanley-McNeil test, "measurements" are required. Therefore, we do not consider the relatively small sample size to be a limitation of our study.

\section{Conclusions}

In this single-center study, we showed that $\triangle \mathrm{CDPV}$ could be more accurate than other methods for assessing fluid responsiveness in patients with septic shock receiving lung protective mechanical ventilation. The $\triangle \mathrm{CDPV}$ also has a high correlation with SVI increase after fluid challenge.

\section{Abbreviations}

$\triangle$ CDPV: Carotid Doppler peak systolic velocity variation; AKl: Acute kidney injury; ARDS: Acute respiratory distress syndrome; AUROC: Area under the receiver operating characteristic curve; BSA: Body surface area; CAD: Coronary artery disease; CVP: Central venous pressure; GEDI: Global end-diastolic index; HR: Heart rate; ICU: Intensive care unit; IVC-d: Inferior vena cava diameter; $\triangle \mathrm{D}$-IVC: Respiratory variation in inferior vena cava diameter; LVOT: Left ventricle outflow tract; MAP: Mean arterial pressure; MaxCDPV: Maximum carotid peak velocity; MinCDPV: Minimum carotid peak velocity; NE: Norepinephrine; NPV: Negative predictive value; PEEP: Positive end-expiratory pressure; PiCCO: Pulse contour cardiac output system; PLR: Passive leg rising; PPV: Pulse pressure variation; PPV*: Positive predictive value; ROC: Receiver operating characteristic; RR: Relative risk; ScvO2: Oxygen saturation at central venous blood; SOFA: Sequential Organ Failure Assessment Score; SVI: Stroke volume index; SW: Stroke volume variation; UO: Urinary output; VTI: Velocity time integral.

Competing interests

The authors declare that they have no competing interests.

\section{Authors' contributions}

MAIE conceived, designed and coordinated the study, performed data collection, statistical analysis, interpretation of data, and drafted the manuscript. JALP performed data collection and provided feedback on the manuscript. JCMM, JLDG, and GAA were involved in drafting the manuscript. All authors read and approved the final manuscript.

\section{Acknowledgements}

We acknowledge Hilario Coronado Magaña, Department Chair of the Intensive Care Unit, for his general support of this work. We would also like to thank Victoria L. Jackson, MLIS, ELS, for her assistance with language editing and the editorial preparation of this manuscript.

\section{Author details}

'Intensive Care Unit, Hospital Civil de Guadalajara "Fray Antonio Alcalde", Hospital 278, El Retiro, Specialties Building, Floor 1, Guadalajara, Jalisco 44280, Mexico. ${ }^{2}$ Centro Universitario de Ciencias de la Salud, Universidad de Guadalajara, Independencia Oriente, 44340 Guadalajara, Jalisco, Mexico. ${ }^{3}$ Departments of Critical Care Medicine, Anesthesiology, and Neurosurgery, Mayo Clinic, 4500 San Pablo Road, Jacksonville, FL, USA

Received: 23 March 2015 Accepted: 7 June 2015

Published online: 26 June 2015

References

1. Hofer CK, Cannesson M (2011) Monitoring fluid responsiveness. Acta Anaesthesiol Taiwan 49:59-65

2. Vincent J (2011) Let's give some fluid and see what happens" versus the "mini-fluid challenge". Anesthesiology 115:455-6

3. Kelm DJ, Perrin JT, Cartin-Ceba R, Gajic O, Schenck L, Kennedy CC (2015) Fluid overload in patients with severe sepsis and septic shock treated with early-goal directed therapy is associated with increased acute need for fluid-related medical interventions and hospital death. Shock 43:68-73

4. Marik PE, Cavallazzi R, Vasu T, Hirani A (2009) Dynamic changes in arterial waveform derived variables and fluid responsiveness in mechanically ventilated patients: a systematic review of the literature. Crit Care Med 37:2642-7

5. Levitov A, Marik PE (2012) Echocardiographic assessment of preload responsiveness in critically ill patients. Cardiol Res Pract 2012:819696

6. Chen C, Kollef MH (2014) Conservative fluid therapy in septic shock: an example of targeted therapeutic minimization. Crit Care 18:481

7. Teboul JL, Monnet X (2008) Prediction of volume responsiveness in critically ill patients with spontaneous breathing activity. Curr Opin Crit Care 14:334-9

8. Dinh VA, Ko HS, Rao R, Bansal RC, Smith DD, Kim TE, Nguyen HB (2012) Measuring cardiac index with a focused cardiac ultrasound examination in the ED. Am J Emerg Med 30:1845-51

9. Mandeville JC, Colebourn CL (2012) Can transthoracic echocardiography be used to predict fluid responsiveness in the critically ill patient? A systematic review. Crit Care Res Pract 2012:513480

10. Huttemann E (2006) Transoesophageal echocardiography in critical care. Minerva Anestesiol 72:891-913

11. Song Y, Kwak YL, Song JW, Kim YJ, Shim JK (2014) Respirophasic carotid artery peak velocity variation as a predictor of fluid responsiveness in mechanically ventilated patients with coronary artery disease. $\mathrm{Br} J$ Anaesth 113:61-6 
12. Monge García Ml, Gil Cano A, Díaz Monrové JC (2009) Brachial artery peak velocity variation to predict fluid responsiveness in mechanically ventilated patients. Crit Care 13(5):R142

13. Dellinger RP, Levy MM, Rhodes A, Annane D, Geriach H, Opal SM, Surviving Sepsis Campaign Guidelines Committee including the Pediatric Subgroup (2013) Surviving sepsis campaign: international guidelines for management of severe sepsis and septic shock: 2012. Crit Care Med 41:580-637

14. Slovut DP, Romero JM, Hannon KM, Dick J, Jaff MR (2008) Detection of common carotid artery stenosis using duplex ultrasonography: a validation study with computed tomographic angiography. J Vasc Surg 51:65-70

15. Michard F, Boussat S, Chemla D, Anguel N, Mercat A, Lecarpentier Y, Richard C, Pinski MR, Teboul JL (2000) Relation between respiratory changes in arterial pulse pressure and fluid responsiveness in septic patients with acute circulatory failure. Am J Respir Crit Care Med 162:134-8

16. Evans D, Ferraioli G, Snellings J, Levitov A (2014) Volume responsiveness in critically ill patients. J Ultrasound Med 33:3-7

17. Hanley JA, Mcneil BJ (1983) A method of comparing the areas under receiver operating characteristic curves derived from the same cases. Radiology 148:839-43

18. Youden WJ (1950) Index for rating diagnostic tests. Cancer 3:32-5

19. Bland JM, Altman DG (1999) Measuring agreement in method comparison studies. Stat Methods Med Res 8:135-60

20. Fleiss JL, Levin B (2003) The measurement of interrater agreement. In: Fleiss $J$ (ed) Statistical Methods for Rates and Proportions, 3rd edn. John Wiley \& Sons, Hoboken

21. Chisholm CB, Dodge WR, Balise RR, Williams SR, Gharahbaghian L, Beraud AS (2013) Focused cardiac ultrasound training: how much is enough? J Emerg Med 44:818-22

22. Yong Y, Wu D, Fernandes V, Kopelen HA, Shimoni S, Nagueh SF, Callahan JD, Bruns DE, Shaw LJ, Quinones MA, Zoghbi WA (2002) Diagnostic accuracy and cost-effectiveness of contrast echocardiography on evaluation of cardiac function in technically very difficult patients in the intensive care unit. Am J Cardiol 89:711-8

23. Feissel M, Michard F, Mangin I, Ruyer O, Faller JP, Teboul JL (2001) Respiratory changes in aortic blood velocity as an indicator of fluid responsiveness in ventilated patients with septic shock. Chest 119:867-73

24. Monnet X, Rienzo M, Osman D, Anguel N, Richard C, Pinsky MR, Teboul JL (2005) Esophageal Doppler monitoring predicts fluid responsiveness in critically ill ventilated patients. Intensive Care Med 31:1195-201

25. Monnet X, Anguel N, Naudin B, Jabot J, Richard C, Teboul JL (2010) Arterial pressure-based cardiac output in septic patients: different accuracy of pulse contour and uncalibrated pressure waveform devices. Crit Care 14:R109

26. Marik PE, Levitov A, Young A, Andrews L (2013) The use of bioreactance and carotid Doppler to determine volume responsiveness and blood flow redistribution following passive leg raising in hemodynamically unstable patients. Chest 143:364-70

27. Dorman T, Breslow MJ, Lipsett PA, Rosenberg JM, Balser JR, Almog Y, Rosenfeld BA (1998) Radial artery pressure monitoring underestimates central arterial pressure during vasopressor therapy in critically ill surgical patients. Crit Care Med 26:1646-9

28. Hong SW, Shim JK, Choi YS, Chun DH, Kim JC, Kim BS, Kwak YL (2009) Predictors of ineffectual radial arterial pressure monitoring in valvular heart surgery. J Heart Valve Dis 18:546-53

29. Nunes T, Ladeira R, Bafi A, de Azevedo LC, Machado FR, Freitas FG (2014) Duration of hemodynamic effects of crystalloids in patients with circulatory shock after initial resuscitation. Ann Intensive Care 4:25

30. Zhang Z, Lu B, Sheng X, Jin N (2011) Accuracy of stroke volume variation in predicting fluid responsiveness: a systematic review and meta-analysis. J Anesth 25:904-16

31. Biais M, Stecken L, Ottolenghi L, Roullet S, Quinart A, Masson F, Sztark F (2011) The ability of pulse pressure variations obtained with CNAP ${ }^{\mathrm{TM}}$ device to predict fluid responsiveness in the operating room. Anesth Analg 113:523-8

32. Solus-Biguenet H, Fleyfel M, Tavernier B, Kipnis E, Onimus J, Robin E, Lebuffe G, Decoene C, Pruvot FR, Vallet B (2006) Non-invasive prediction of fluid responsiveness during major hepatic surgery. Br J Anaesth 97:808-16

33. Serpa Neto A, Cardoso SO, Manetta JA, Pereira VG, Esposito DC, Pasqualicci Mde O, Damasceno MC, Schultz MJ (2012) Association between use of lung-protective ventilation with lower tidal volumes and clinical outcomes among patients without acute respiratory distress syndrome: a meta-analysis. JAMA 308:1651-9
34. Michard F, Teboul JL, Richard C (2003) Influence of tidal volume on stroke volume variation. Does it really matter? Intensive Care Med 29:1613

35. Shim JK, Song JW, Song Y, Kim JH, Kang HM, Kwak YL (2014) Pulse pressure variation is not a valid predictor of fluid responsiveness in patients with elevated left ventricular filling pressure. J Crit Care 29:987-91

36. Cameli M, Bigio E, Lisi M, Righini FM, Galderisi M, Franchi F, Scolletta S, Mondillo S (2014) Relationship between pulse pressure variation and echocardiographic indices of left ventricular filling pressure in critically ill patients. Clin Physiol Funct Imaging. 2014 Jun 5. [Epub ahead of print]

37. Caillard A, Gayat E, Tantot A, Dubreuil G, M'Bakulu E, Madadaki C, Bart F, Bresson D, Froelich S, Mebazaa A, Vallée F (2015) Comparison of cardiac output measured by oesophageal Doppler ultrasonography or pulse pressure contour wave analysis. Br J Anaesth. [Epub ahead of print]

38. Huber W, Koenig J, Mair S, Schuster T, Saugel B, Eyer F, Phillip V, Schultheiss C, Thies P, Mayr U, Einwächter H, Treiber M, Hoellthaler J, Schmid RM (2015) Predictors of the accuracy of pulse-contour cardiac index and suggestion of a calibration-index: a prospective evaluation and validation study. BMC Anesthesiol 15:45

\section{Submit your manuscript to a SpringerOpen ${ }^{\mathcal{D}}$ journal and benefit from:}

- Convenient online submission

Rigorous peer review

- Immediate publication on acceptance

- Open access: articles freely available online

- High visibility within the field

- Retaining the copyright to your article

Submit your next manuscript at $>$ springeropen.com 